\title{
Vorbei mit der Freiheit in der Fortbildung?
}

\section{Christian Weber}

Präsident der Fortbildungskommission Schweizerische Gesellschaft für Psychiatrie und Psychotherapie SGPP

In seinem Artikel zum Sinn und Unsinn des neuen psychiatrischen Fortbildungsprogramms [1] beklagt der Autor den Verlust der «letzten Freiheit» anlässlich der Streichung der 10 Stunden «freier Fortbildung» aus dem alten Programm und plädiert für mehr Fantasie, Kreativität und Freiheit in der Fortbildung.

Diesem Grundtenor stimmen die angesprochene Fortbildungskommission wie auch die Verantwortlichen des SIWF nur zu, und sie haben in letzter Zeit einiges getan, um die Fortbildungspflicht zu liberalisieren. Zweifellos kann und darf die Fortbildung nicht allein von der Politik bestimmt werden, weder Reglemente noch das blosse Sammeln von Credits, weder Normierung und Zertifizierung noch das beste Q-Monitoring sind Garanten für eine qualitativ ausreichende Fortbildung des einzelnen Arztes.

Gerade deswegen heisst es schon im ersten Abschnitt des Fortbildungsprogramms der SGPP «grundsätzlich ist die ärztliche Fortbildung ein kontinuierlicher und persönlicher Entwicklungsprozess, welcher essentiell auf Eigenverantwortung gründet. Es gehört zur ärztlichen Ethik, diesen Prozess während der ganzen Dauer der Praxistätigkeit voranzutreiben». Und weiter: «Fortbildungspflichtige Ärzte absolvieren dasjenige Fortbildungsprogramm, das ihrer aktuellen Berufstätigkeit entspricht.» Das bedeutet, dass auch Mehrfachtitelträger nur ein einziges Fortbildungsdiplom erwerben müssen. Ferner wurde neu eingeführt, dass jede für einen Schwerpunkt absolvierte Fortbildung auch als fachspezifische Kernfortbildung für den Haupttitel zählt.

\section{Bei Lichte besehen, muss angesichts der Menge von Fortbildungsmöglichkeiten und individueller Gestaltungsfreiheit doch eher von der Qual der Wahl als vom Verlust der Freiheit gesprochen werden.}

Korrespondenz: Dr. med. Christian Weber Chefarzt

Clienia Schlössli AG Psychiatriezentrum Männedorf Bergstrasse 34 CH-8708 Männedorf Tel. 0438433200 Fax 0438433211
Wer das geschmähte und zugegeben etwas aufwendig zu lesende Fortbildungsprogramm der SGPP genau studiert, entdeckt im definierten Rahmen viele Anregungen und einen weiten Raum für eine formal und inhaltlich ausgesprochen vielseitige Fortbildungsgestaltung. Dabei reicht das Spektrum von Supervision, Intervision, Qualitätszirkelarbeit und Selbsterfahrung über alle Arten von theoretischer Fortbildung bis zu Lehr- und Forschungstätigkeit. Mit der neuen Fortbildungsordnung (FBO) des SIWF wird auch der Blick über den Tellerrand des eigenen Fachgebie- tes hinaus bewusst gefördert, indem Veranstaltungen von anderen Fachgesellschaften als erweiterte Fortbildung angerechnet werden können, und das neue System von Kernfortbildung und erweiterter Fortbildung bietet eine Vielzahl von Kombinationsmöglichkeiten. Bei Lichte besehen, muss angesichts der Menge von Fortbildungsmöglichkeiten und individueller Gestaltungsfreiheit doch eher von der Qual der Wahl als vom Verlust der Freiheit gesprochen werden.

Angenommen, unser Kollege beschäftigt sich, wie im Artikel ausgeführt, angesichts seiner Patienten mit existentiellen Fragen nach dem Lebenssinn und Lebensglück und er würde beispielsweise eine (von der SGPP nicht-creditierte) Vorlesung der philosophischen Fakultät über das «gelingende Leben» besuchen und dies in seinem Fortbildungsprotokoll notieren, und angenommen, er müsste nun zufällig in der Stichprobenkontrolle sein Protokoll vorlegen - er würde von der Fortbildungskommission gewiss ein anerkennendes Brieflein für seine differenzierte und vielseitige Fortbildung erhalten, zumindest solange auch die klassische Kernfortbildung mit ihren jährlichen 25 Credits sichergestellt ist. Würde hingegen seine gesamte individuelle Fortbildung aus lauter philosophischen und anderen lebenskundlichen Veranstaltungen bestehen, erhielte er ebenso gewiss ein mahnendes Schreiben, in welchem ihm mitgeteilt würde, dass ihm das Fortbildungsdiplom leider nicht erteilt werden könne mit entsprechenden Verbesserungshinweisen.

In diesem Zusammenhang sind aus der Tätigkeit der Fortbildungskommission der SGPP die folgenden beiden Erfahrungen interessant:

- Die Durchsicht der Stichproben zeigt regelmässig, dass die grosse Mehrheit der Fachkollegen die Anzahl der geforderten Fortbildungsstunden bei weitem übertrifft - da kann man nur gratulieren.

- Zum anderen dominieren bei den Anfragen zu Fortbildungsanerkennungen diejenigen aus der ängstlich-besorgten Ecke - da kann man nicht genug auf das oberste Prinzip der eigenverantwortlichen Gestaltung der Fortbildungstätigkeit jenseits übertriebener Credithörigkeit hinweisen.

Das SIWF und die Fachgesellschaften legen für die Fortbildungserfüllung einen hilfreichen Rahmen fest, welcher durch den einzelnen Arzt individuell, kreativ und in aller Freiheit auszugestalten ist.

1 Dudle U. Vorbei mit der Freiheit. Sinn und Unsinn in der neuen psychiatrischen Fortbildungsverordnung. Schweiz Ärztezeitung. 2010;91(43):1704-5. 